\title{
Queratoquiste Odontogénico en un Paciente con Displasia Cleidocraneal: Reporte de un Caso
}

\author{
Odontogenic Keratocyst in a Patient with Cleidocranial Dysplasia: Case Report
}

\author{
Gonzalo Martinovic Guzmán; Miguel Vergara Iturriaga; \\ Juan Pablo Alister Herdener ${ }^{3} \&$ Carlos Manterola ${ }^{4}$
}

\begin{abstract}
MARTINOVIC, G. G.; VERGARA, M. I.; ALISTER, J. P. H. \& MANTEROLA, C. Queratoquiste odontogénico en un paciente con displasia cleidocraneal: Reporte de un caso. Int. J. Odontostomat., 13(2):189-194, 2019.

RESUMEN: La displasia cleidocraneal (DCC), es un trastorno autosómico dominante poco común, que involucra principalmente a los huesos que se osifican por vía membranosa; afectando el cierre de fontanelas craneales y el desarrollo de las clavículas, además de anomalías dentales y vertebrales. El objetivo de este manuscrito fue reportar el caso de una paciente con DCC que presentó un queratoquiste odontogénico (QQO) intrasinusal. Presentamos el caso de una paciente de 81 años, diagnosticada en su niñez con DDC, que consultó por un desajuste protésico y molestias en relación a la zona del seno maxilar derecho. Clínicamente se observó desajuste de la prótesis y aumento de volumen de márgenes poco definidos en la zona maxilar derecha, color rosa coral; que se extendía por todo el margen hemimaxilar derecho hasta el fondo de vestíbulo; doloroso a la palpación, con un mes de evolución. Se solicitó CBCT, con el que se pudo verificar la presencia de un desarrollo tumoral de contenido similar a dentículos, ubicado en la totalidad del seno maxilar derecho; extendiéndose hasta el piso de la cavidad nasal y orbitaria. Se estableció la hipótesis diagnóstica de "odontoma compuesto". Se le intervino quirúrgicamente, bajo anestesia general, realizándose una excisión de la lesión; la que era de márgenes definidos, con cambios de coloración en tonos oscuros, con la inclusión de tres piezas dentarias; de aspecto maligno. Se logró enucleación completa, dejando remanente óseo limpio. La pieza fue enviada a estudio histopatológico. En informe histopatológico, describió la presencia de una lesion quistica con pared compatible con queratoquiste.
\end{abstract}

PALABRAS CLAVE: displasia cleidocraneal, queratoquiste, dientes supernumerarios.

\section{INTRODUCCIÓN}

Las displasias esqueléticas son enfermedades poco frecuentes, de origen genético, cuyo diagnóstico se basa casi exclusivamente en elementos clínicos y radiológicos. Son de evolución crónica, muchas veces progresivas, y no existe tratamiento curativo (Krakow, 2015). Una de ellas, es la displasia cleidocraneal (DCC), la que puede presentarse desde una forma clásica, con retraso de cierre de las suturas craneales, clavículas hipoplásicas o aplásicas y anormalidades dentarias; a una forma leve en que sólo se presentén alteraciones dentarias (Golan et al., 2000).

El $94 \%$ de pacientes con DCC presentan dientes supernumerarios, retraso en la exfoliacion de dientes temporales y alteración en la erupción de los dientes permanentes (Golan et al., 2003; Bufalino et al., 2012). Además, son más propensos a desarrollar una mordida invertida y formar quistes alrededor de los dientes supernumerarios (McNamara et al., 1999). También se ha descrito retrusión del tercio medio facial y prognatismo mandibular acompañado de baja estatura (Farrow et al., 2018).

El tratamiento odontológico de estos casos, involucra la desinclusión temprana de los dientes retenidos para prevenir la aparición de patología concomitante a las inclusiones dentarias. En general más del $90 \%$ de los supernumerarios se ubican en maxilar; y solo un $25 \%$ de estos, erupcionan por lo

\footnotetext{
${ }^{1}$ Cirujano Maxilofacial, Hospital Militar Santiago, Programa de Magíster en Ciencias Médicas, Universidad de La Frontera, Temuco, Chile. ${ }^{2}$ Cirujano Dentista.

${ }^{3}$ Facultad de Odontología, Universidad de La Frontera, Temuco, Chile.

${ }^{4}$ Departamento de Cirugía y Centro de Estudios Morfológicos y Quirúrgicos (CEMyQ), Universidad de La Frontera, Temuco, Chile.
} 
que comúnmente permanecen incluídos y requiren extracción (Klein et al., 2013). Una alta incidencia de dientes supernumerarios es encontrada en pacientes con fisura palatina, así como también en más de 20 síndromes, siendo los más comunes la DCC y el síndrome de Gardner (Moore et al., 2002).

El queratoquiste odontogénico ( $Q Q O)$, fue reconocido como tumor luego de observar su progresión neoplásica. Esta lesión es única entre los quistes odontogénicos debido a su aspecto microscópico patognomónico, conducta agresiva y alta tasa de recurrencia (Wright \& Vered, 2017). La OMS los reclasificó como lesiones quísticas, argumentando que una verdadera neoplasia no regresa espontáneamente a la descompresión (Wright \& Vered).

El objetivo de este manuscrito fue reportar el caso de una paciente con DCC que presentó un $\mathrm{QQO}$ intrasinusal.

\section{REPORTE DEL CASO}

Paciente de sexo femenino, 81 años, con DCC diagnosticado en su infancia. Consultó por desajuste de la prótesis removible maxilar y dolor de un mes de evolución en la región maxilar derecha. Al examen clínico, se verificó desdentada total superior e inferior, con una leve asimetría facial y aumento de volumen de la región geniana derecha; y ocupación parcial del fondo del vestíbulo hemimaxilar derecho, caracterizado por consistencia firme a la palpación (Figs. 1a,b).

Se solicitó un Cone Beam CT bimaxilar, con el que se logró constatar la existencia de una masa quística de contenido similar a dentículos alojados en la casi totalidad del seno maxilar derecho (Figs. 2a-c).

Con estas imágenes, se planteó el diagnóstico de odontoma compuesto; a pesar de ser ésta una lesión habitualmente indolora y de localización más frecuente en sector anterior.

Se decidió intervenir quirúrgicamente, para lo cual se tomaron las medidas de la lesión, como parte de la planificación de la cirugía (Figs. 3a-c).

La paciente fue operada en el pabellón central del Hospital Militar de Santiago, Chile; en Noviembre del año 2016. En esta oportunidad, bajo anestesia general y a través de acceso intraoral, se realizó la enucleación total de la masa quística con márgenes de seguridad; extirpando una masa irregular de $4 \mathrm{~cm}$, de márgenes mal definidos, coloración oscura, heterogénea, y no corticalizado con la inclusión de 3 piezas dentarias. Además, se verificó la presencia de queratina con signos de infección.

La masa invadía casi la totalidad del espacio comprendido entre el margen alveolar y el piso de la órbita derecha (Figs. 4a-f). Por lo que se decidió tomar una conducta agresiva y eliminar la lesión totalmente. Las muestras obtenidas fueron enviadas a cultivo corriente y análisis histopatológico.

Los resultados del estudio hisptopatológico fueron: Muestra constituida por tres piezas dentarias color marfil, superficie algo irregular, memelonada, con raíz de superficie anfractuosa, escasas adherencias a material hemático coagulado. Asociado por separado se reconocen paredes de formación quística, en partes rotas paredes fi-
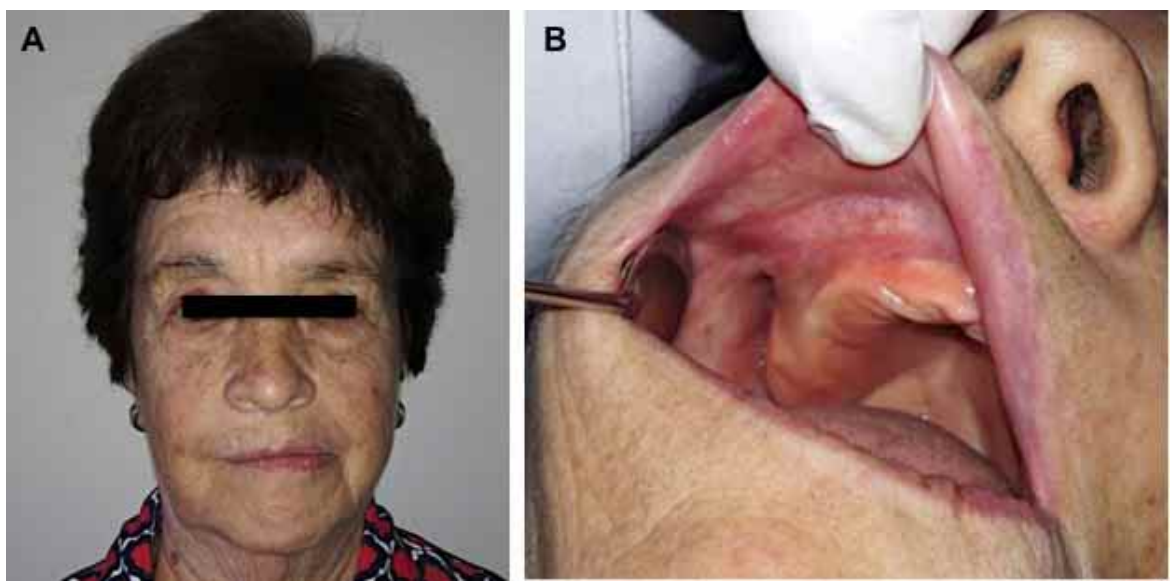

Fig. 1A y B. Fotografías clínicas preoperatorias de la paciente, que permiten evidenciar el maxilar edéntulo y el aumento de volumen con fondo de vestíbulo parcialmente ocupado. 

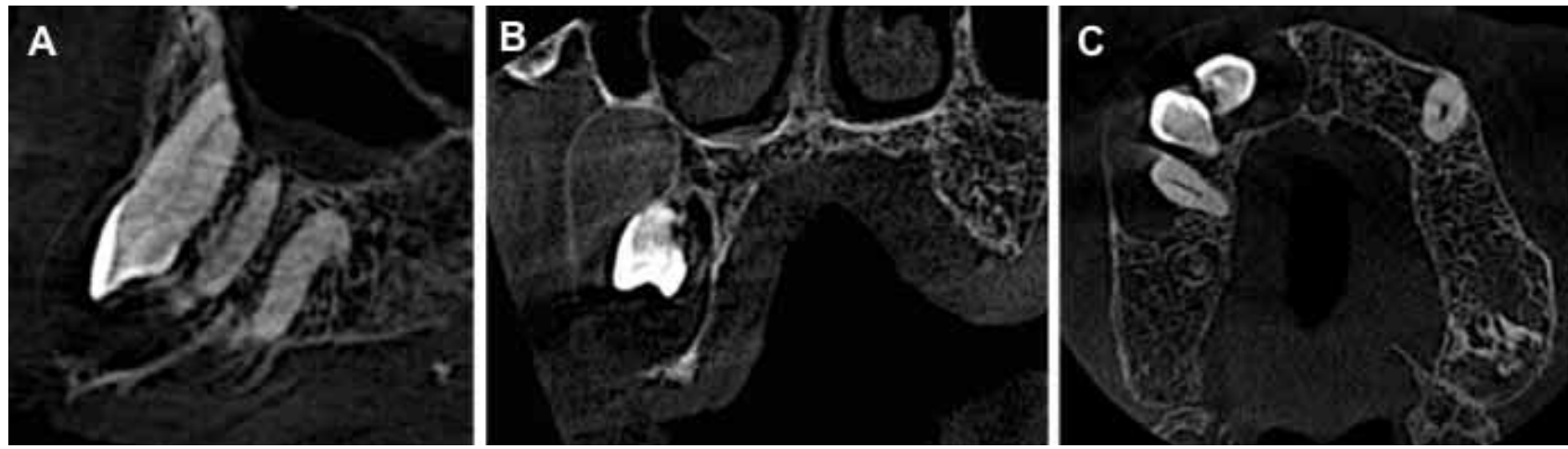

Fig. 2A, B y C. Tomografía computarizada inicial. Cortes transversal, coronal y sagital respectivamente. Se puede apreciar los limites del quiste, su relación con el piso de la órbita, el seno maxilar y la inclusión de multiples piezas.

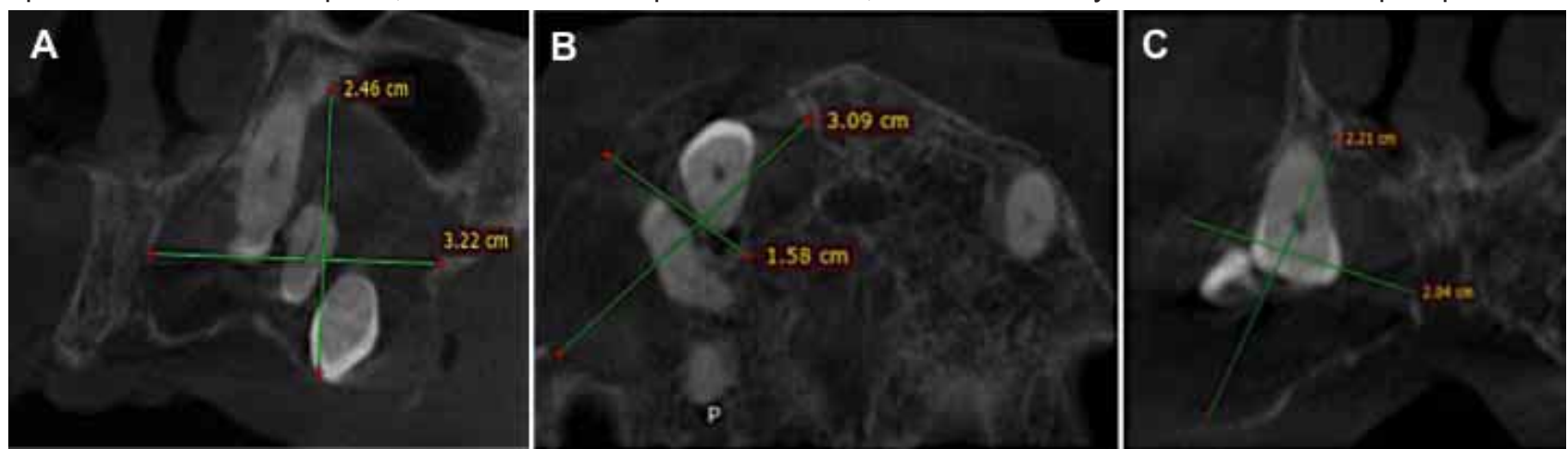

Fig. 3A, B y C. Tomografía computarizada, en la que se constata las mediciones de la lesión quística en cortes transversal, coronal y sagital respectivamente.
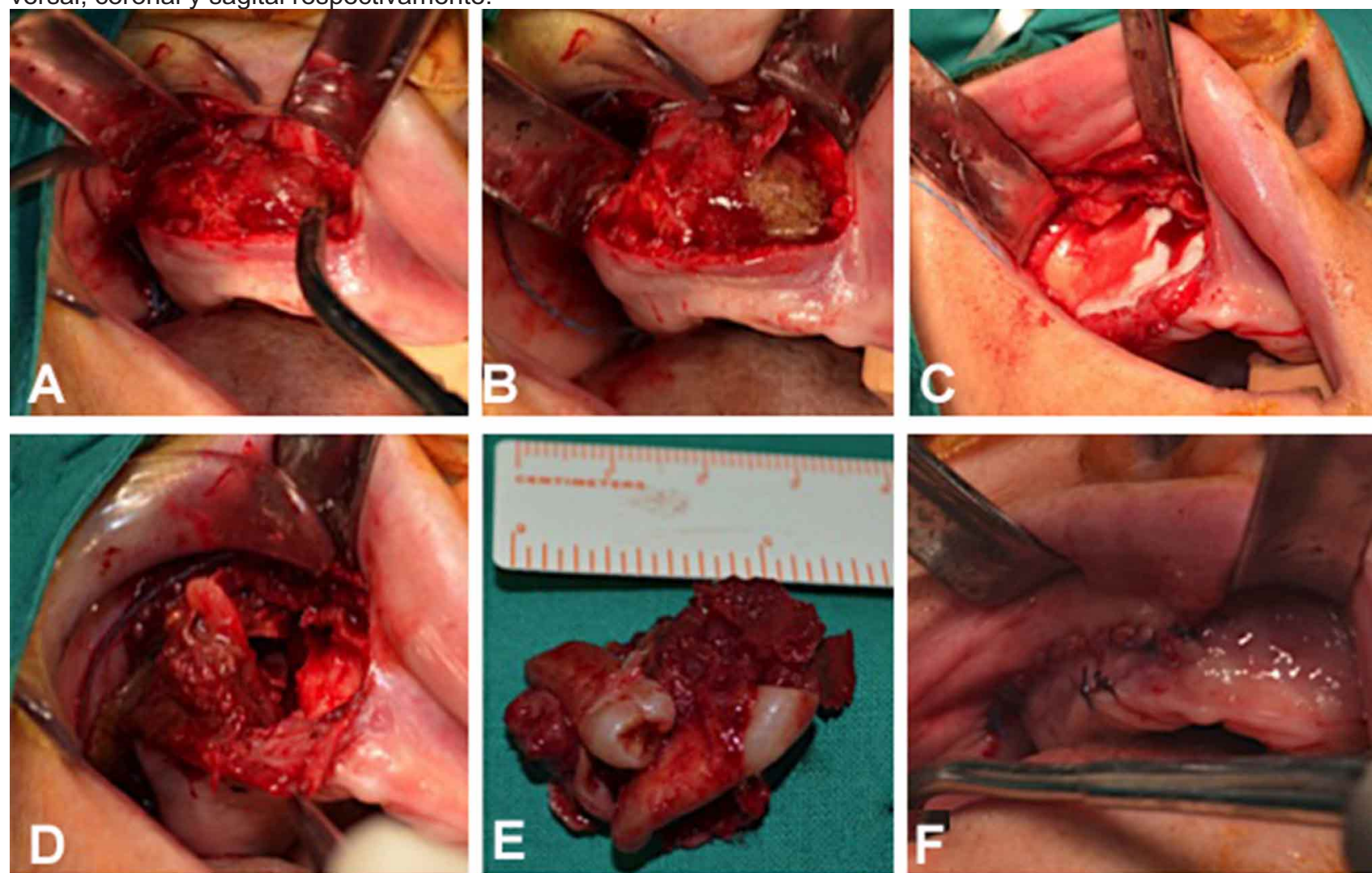

Fig. 4A, B, C, D, E y F. Secuencia quirúrgica. Tejidos blandos y calcificados (D). Pieza quirúrgica (E). Hemostasia y cierre (F). 
nas, anfractuosas, color pardo grisáseo, la cual alcanza a $3 \times 2 \mathrm{~cm}$ en eje mayor y es de consistencia elástica. Su superficie interna es algo trabeculada, pardo grisácea. La conclusión del estudio histopatológico fue: "aspecto histológico concordante con pared de queratoquiste, asociado a tres piezas dentarias de histoarquitectura preservada; sin evidencias de elementos con caracteres de malignidad".

Tras un seguimiento de dos años, la paciente se encuentra en buenas condiciones generales, asintomatica y sin signos de recidiva de la lesion.

\section{DISCUSIÓN}

Se realizó una búsqueda sistemática de la literatura relacionada en la base de datos MEDLINE, utilizando el buscador PubMed, con los términos "cleidocranial dysplasia"[MeSH Terms], "Odontogenic Cyst" [MeSH Terms], "Odontogenic Cysts", Keratocysts, Keratocyst, Odontoma, Impacted Tooth, Teeth, Impacted, Impacted Teeth. Se utilizaron además los operadores booleanos AND y OR para generar las estrategia de búsqueda; a las que se aplicaron posteriormente los filtros: estudios en humanos e idioma inglés. Esto, permitió recuperar 133 estudios relacionados; de los cuales, tras la lectura de los títulos; luego de los resúmenes; y por último, de los extensos; se descartaron 113. Por ende, esta discusión se realizó con base en los 20 artículos finalmente seleccionados.

La DCC es un síndrome que presenta variadas anomalías a nivel maxilofacial, dentro de las cuales la presencia de piezas supernumerarias es una situación común (Golan et al., 2003; Bufalino et al.). La paciente fue diagnosticada en su niñez con este síndrome; y al consultar, se realizó una hipótesis diagnóstica de odontoma compuesto dado a la presencia de dientes en la lesión.

Los odontomas compuestos son considerados malformaciones del desarrollo del tejido dentario. Se forman de esmalte y dentina; y pueden tener cantidades variables de cemento y pulpa. Tambien se describen como un conglomerado de pequeñas estructuras similares a dientes (Bordini et al., 2008). Se presenta con mayor frecuenca en mujeres y la edad de aparición es alrededor de los 23 años. Se ubican de preferencia en el maxilar superior, generalmente en la región anterior. Se asocian a dientes no erupcionados en la mayoría de los casos. Radiográficamente, se observan como una imagen radiopaca irregular, compuesta por múltiples radiopacidades, rodeado por un halo radiolúcido. Histológicamente esta lesión está compuesta por varios dentículos pequeños, de diferentes grados de diferenciación (Bereket et al., 2015).

El odontoma compuesto es de crecimiento lento y asintomático, por ello son generalmente diagnosticados mediante exámenes radiográficos de rutina, alrededor de la segunda y tercera década de vida (Amado Cuesta et al., 2003). Si no aparecen signos ni síntomas, esta lesión puede permanecer en el hueso muchos años sin producir manifestaciones clínicas, tales como formación de quistes dentígenos o quiste odontogénico epitelial calcificante (Fernandes et al., 2005). El tratamiento de elección para estos casos es la enucleación simple de la lesión, siendo un tratamiento conservador (Boffano et al., 2012).

Por su parte los QQO, se caracterizan clínicamente por ser lesiones únicas, y ubicarse frecuentemente en la rama y ángulo mandibular. Por lo general son asintomáticos y se descubren en exámenes radiográficos de rutina. En lesiones de mayor tamaño, pueden desplazar la posición de algunos dientes, aparecer inflamación debido a la expansión de la cortical (por drenaje espontáneo del contenido quístico); y presentarse con dolor en casos de infección (Maurette et al., 2006). Se ha descrito que el 38 $\%$ de los casos se presentan con inflamación, dolor y descarga. (Fernandes et al.). Se observa mayoritariamente en mujeres, entre los 20 y 40 años (Kolokythas et al., 2007).

El aspecto radiográfico de éstas lesiones es variable, pudiendo presentarse como un área radiolúcida unilocular o multilocular, generalmente con límites bien definidos, con o sin la presencia de un aro esclerótico periférico (Omura et al., 1997). Existe evidencia respecto que las lesiones multiloculares se encuentran en sujetos de mayor edad, sugieriendo la posibilidad que las uniloculares pudiesen progresar a multiloculares. Los QQO que afectan al maxilar, son más frecuentes en individuos jóvenes, y son habitualmente uniloculares (MacDonald-Jankowski \& Li, 2010).

Los queratoquistes presentan una tasa de recurrencia promedio del $25 \%$. Debido a esto, es necesario realizar resecciones con márgenes de seguridad y un riguroso seguimiento post operatorio clí- 
nico y radiográfico. En ocasiones estos quistes se asocian a dientes incluidos o impactados. La evidencia apoya la tesitura que pueden presentarse hasta en un $27 \%$ de los casos; y que esta patología es 5 veces más frecuente en mandíbula que en maxilar; y que en el $30 \%$ de estos casos, afecta más de un área anatómica, generalmente cuerpo, ángulo y rama mandibular. Se ha observado que QQO en relación a tejido dentario, presentan mayor riesgo de recurrencia (Sánchez-Burgos et al., 2014).

En este caso, durante el acto quirúrgico se detectó que la lesión tenía un tamaño mayor al detectado imagenológicamente, con límites poco claros y características de malignidad; por lo cual fue necesario realizar una resección más agresiva y un curetaje de la zona, para lograr eliminar todo el tejido que conformaba esta lesión. La histología fue de queratoquiste; por ende, se trató de un QQO intrasinusal asociado a dientes incluidos.

En una investigación realizada por Mortazavi \& Baharvand (2016) se obtuvo que las lesiones más frecuentes asociadas a dientes impactados eran el ameloblastoba uniquístico, fibrodontoma ameloblástico, tumor odontogénico adenomatoide, odontoma y quistes dentígenos. En contraste, las de menor prevalencia de asociación fueron los quistes odontogénicos calcificantes y queratoquiste. El diente más frecuente que presentó lesiones fue el tercer molar madibular, excepto para tumores odontogénicos adenomatoides, quistes odontogénicos calcificantes y odontomas compuestos, que tuvieron de preferencia dientes del sector anterior maxilar (Mortazavi \& Baharvand). Este mismo estudio reveló que el quiste odontogénico calcificante, ameloblastoma, queratoquiste odontogénico y el tumor odontogénico epitelial calcificante, tienen dos picos temporales de aparición: en la juventud, y edad mediana o mayor (Mortazavi \& Baharvand).

Es imprescindible tomar en cuenta que la presencia de dientes supernumerarios pueden propiciar la formación de otras patologías como quistes (McNamara et al.); y que existe evidencia respecto que la mayoría de los QQO se asocian a dientes no erupcionados (MacDonald-Jankowski \& Li).

Es necesario prestar principal atención a las anomalías producidas por la DCC, que pueden conllevar o ser un factor predisponente para el desarrollo de tumores o quistes en la zona de los huesos maxilares.
MARTINOVIC, G. G.; VERGARA, M. I.; ALISTER, J. P. H. \& MANTEROLA, C. Odontogenic keratocyst in a patient with cleidocranial dysplasia: Case Report. Int. J. Odontostomat., 13(2):189-194, 2019.

ABSTRACT: Cleidocranial dysplasia (CCD) is an uncommon autosomal dominant disorder that mainly involves bones that ossify via the membrane, affecting the closure of cranial fontanels and the development of the clavicles, as well as presenting dental and vertebral anomalies. The aim of this manuscritpt was to report a case of a patient with CCD who presented an intrasinusal odontogenic keratocyst.We present an 81-year-old female patient, diagnosed with this syndrome in childhood, who comes to our service for a prosthetic misalignment and discomfort of the right maxillary sinus area. Clinically, there was a mismatch of the prosthesis and an increase in the volume of undefined margins under it, coral pink color, which extended all over the right hemimaxillary margin to the bottom of the vestibule, painful on palpation, with a one month evolution. A CBCT was requested, which revealed the presence of a tumor development with content similar to denticles, located in the entire right maxillary sinus, extending to the floor of the nasal and orbital cavity. The diagnostic hypothesis of "compound odontoma" was established. The patient was operated on in the central ward, under general anesthesia performing the excisional biopsy of the lesion, which showed changes in coloration in dark tones, with defined edges, with the inclusion of three teeth showing malignancy aspects. Complete enucleation was achieved, leaving tumor-free clean bone remnant. In a histopathological report, the presence of a keratocyst wall was described, which is not very compatible given the appearance of the lesion, the presence of the dental pieces included in it, and the behavior of the lesion.

KEY WORDS: "Cleidocranial dysplasia"[MeSH Terms], "Odontogenic Cyst" [MeSH Terms], "Odontogenic Cysts", Keratocysts, Keratocyst, Odontoma, Impacted Tooth, Teeth, Impacted, Impacted Teeth.

\section{REFERENCIAS BIBLIOGRÁFICAS}

Amado Cuesta, S.; Gargallo Albiol, J.; Berini Aytés, L. \& Gay Escoda, C. Review of 61 cases of odontoma. Presentation of an erupted complex odontoma. Med. Oral, 8(5):366-73, 2003.

Bereket, C.; Çakır-Özkan, N.; Sener, I.; Bulut, E. \& Tek, M. Complex and compound odontomas: Analysis of 69 cases and a rare case of erupted compound odontoma. Niger. J. Clin. Pract., 18(6):726-30, 2015.

Boffano, P.; Zavattero, E.; Roccia, F. \& Gallesio, C. Complex and compound odontomas. J. Craniofac. Surg., 23(3):685-8, 2012.

Bordini, J. Jr.; Contar, C. M.; Sarot, J. R.; Fernandes, A. \& Machado, M. A. Multiple compound odontomas in the jaw: case report and analysis of the literature. J. Oral Maxillofac. Surg., 66(12):2617-20, 2008. 
Bufalino, A.; Paranaíba, L. M.; Gouvêa, A. F.; Gueiros, L. A.; Martelli-Júnior, H.; Junior, J. J.; Lopes, M. A.; Graner, E.; De Almeida, O. P.; Vargas, P. A.; et al. Cleidocranial dysplasia: oral features and genetic analysis of 11 patients. Oral Dis., 18(2):184-90, 2012.

Farrow, E.; Nicot, R.; Wiss, A.; Laborde, A. \& Ferri, J. Cleidocranial dysplasia: a review of clinical, radiological, genetic implications and a guidelines proposal. J. Craniofac. Surg., 29(2):382-9, 2018.

Fernandes, A. M.; Duarte, E. C.; Pimenta, F. J.; Souza, L. N.; Santos, V. R.; Mesquita, R. A. \& de Aguiar, M. C. Odontogenic tumors: a study of 340 cases in a Brazilian population. J. Oral Pathol. Med., 34(10):583-7, 2005

Golan, I.; Baumert, U.; Hrala, B. P. \& Müssig, D. Dentomaxillofacial variability of cleidocranial dysplasia: clinicoradiological presentation and systematic review. Dentomaxillofac. Radiol., 32(6):347-54, 2003.

Golan, I.; Preising, M.; Wagener, H.; Baumert, U.; Niederdellmann, H.; Lorenz, B. \& Müssig, D. A novel missense mutation of the CBFA1 gene in a family with cleidocranial dysplasia (CCD) and variable expressivity. J. Craniofac. Genet. Dev. Biol., 20(3):113-20, 2000.

Klein, O. D.; Oberoi, S.; Huysseune, A.; Hovorakova, M.; Peterka, M. \& Peterkova, R. Developmental disorders of the dentition: an update. Am. J. Med. Genet. C Semin. Med. Genet., 163C(4):318-32, 2013.

Kolokythas, A.; Fernandes, R. P.; Pazoki, A. \& Ord, R. sA. Odontogenic keratocyst: to decompress or not to decompress? A comparative study of decompression and enucleation versus resection/peripheral ostectomy. J. Oral Maxillofac. Surg., 65(4):640-4, 2007.

Krakow, D. Skeletal dysplasias. Clin. Perinatol., 42(2):301-19, 2015.

MacDonald-Jankowski, D. S. \& Li, T. K. Keratocystic odontogenic tumour in a Hong Kong community: the clinical and radiological features. Dentomaxillofac. Radiol., 39(3):167-75, 2010.

Maurette, P. E.; Jorge, J. \& de Moraes, M. Conservative treatment protocol of odontogenic keratocyst: a preliminary study. J. Oral Maxillofac. Surg., 64(3):379-83, 2006.

McNamara, C. M.; O'Riordan, B. C.; Blake, M. \& Sandy, J. R. Cleidocranial dysplasia: radiological appearances on dental panoramic radiography. Dentomaxillofac. Radiol., 28(2):89-97, 1999.

Moore, S. R.; Wilson, D. F. \& Kibble, J. Sequential development of multiple supernumerary teeth in the mandibular premolar region -- a radiographic case report. Int. J. Paediatr. Dent., 12(2):143-5, 2002

Mortazavi, H. \& Baharvand, M. Jaw lesions associated with impacted tooth: A radiographic diagnostic guide. Imaging Sci. Dent., 46(3):147-57, 2016.

Omura, S.; Kawabe, R.; Kobayashi, S. \& Mizuki, N. Odontogenic keratocyst appearing as a soap-bubble or honeycomb radiolucency: Report of a case. J. Oral Maxillofac. Surg., 55(2):185-9, 1997.

Sánchez-Burgos, R.; González-Martín-Moro, J.; Pérez-Fernández, E. \& Burgueño-García, M. Clinical, radiological and therapeutic features of keratocystic odontogenic tumours: a study over a decade. J. Clin. Exp. Dent., 6(3):e259-64, 2014.

Wright, J. M. \& Vered, M. Update from the 4th Edition of the World Health Organization Classification of Head and Neck Tumours: Odontogenic and Maxillofacial Bone Tumors. Head Neck Pathol., 11(1):68-77, 2017.
Dirección para correspondencia:

Gonzalo Martinovic Guzmán

Hospital Militar Santiago - Chile.

Av. Alcalde Fernando Castillo Velasco 9100

La Reina, Región Metropolitana

Santiago

CHILE

E-mail: drmartinovicg@gmail.com

Recibido : 30-01-2018

Aceptado: 03-03-2019 